\title{
RARE CASE REPORTS OF COMPLETE UTERINE SEPTUM WITH LONGITUDINAL VAGINAL SEPTUM PRESENTING AS DIDELPHIC/ UNICORNUATE UTERUS
}

\author{
B. Kalpana1, Richa Gupta ${ }^{2}$
}

${ }^{1}$ HOD (Consultant), Department of Reproductive Medicine, Guru Hospital, Madurai. 2Junior Consultant, Department of Reproductive Medicine, Guru Hospital, Madurai.

HOW TO CITE THIS ARTICLE: Kalpana B, Gupta R. Rare case reports of complete uterine septum with longitudinal vaginal septum presenting as didelphic/ unicornuate uterus. J. Evolution Med. Dent. Sci. 2017;6(94):6920-6922, D0I: 10.14260/jemds/2017/1501

\section{PRESENTATION OF CASE}

Müllerian duct anomalies (MDA) comprise of a heterogeneous group of genital malformations accounting for an incidence of $3 \%-4 \%$ in the general female population. Use of improved imaging modalities such as 3D transvaginal ultrasound (TVUS) and magnetic resonance imaging (MRI) has helped clinicians to better identify and characterise such anomalies.[1] Septate uterus with cervical duplication and longitudinal vaginal septum is a rare Müllerian anomaly, hence its true incidence is not known.

We report 2 cases of this rare anomaly.

\section{CASE REPORT 1}

A 23 years old female, married for 1.5 years presented to us with complaints of primary infertility. Her menstrual cycles were regular (3/30) with dysmenorrhea and dyspareunia. Per speculum examination revealed a longitudinal vaginal septum and 2 cervices. Hysterosalpingogram (HSG) (Fig. 1) and TVUS suggested 2 uterine cavities with 2 cervices. A right complex ovarian cyst was also seen on TVUS. MRI confirmed the diagnosis as uterine didelphys with adenomyosis with right ovarian chocolate cyst with left PCOD. There was no evidence of renal anomaly. We proceeded with hysterolaparoscopy.

Laparoscopy revealed a broad single smooth uterine fundus with bilateral normal tubes. Right ovary had a chocolate cyst for which cystectomy was done and left ovary was normal. Hysteroscopy revealed a complete uterine septum extending till external os and a longitudinal vaginal septum as shown in Fig. 2.

\section{CASE REPORT 2}

A 20 years old female, married for 3 years presented to our clinic as case of primary infertility. She had regular menstrual cycles with no dysmenorrhea or dyspareunia. She had undergone laparotomy with right salpingo-oophorectomy for right chocolate cyst at the age of 16 years. Her HSG report was suggestive of left unicornuate uterus as shown in Fig. 3. She had a USG report suggestive of unicornuate uterus with absent right kidney and a chocolate cyst in left ovary. She also had an MRI report, which suggested a possibility of Uterus Didelphys with left ovarian chocolate cyst. As there was a variation between HSG and MRI impression, we proceeded with hysteroscopy.

'Financial or Other Competing Interest': None.

Submission 30-10-2017, Peer Review 23-11-2017,

Acceptance 01-12-2017, Published 18-12-2017.

Corresponding Author:

Dr. B. Kalpana,

\#4/120 F, Pandikovil Ring Road,

Madurai-625107,

Tamilnadu.

E-mail: balamurugan_kalpana@rediffmail.com

DOI: $10.14260 /$ jemds $/ 2017 / 1501$
Hysteroscopy revealed a small uterine cavity deviated to the left side, left ostium was seen but right ostium was not visualised suggestive of left unicornuate uterus. On laparoscopy, the uterine fundus was smooth and broad. Right tube and ovary were absent. Left tube was normal and left ovary had Polycystic Ovarian Disease (PCOD).

In view of these inconclusive findings, we probed to look for another opening in the perineum and found a small hole of $0.2 \mathrm{~cm}$ on right side of vulval outlet with mucus and pus coming out of it. We dilated the opening and let the pus out. We introduced the hysteroscope through the same opening and visualised the right half of vagina and cervix and gently proceeded with hysteroscopy. The uterine cavity was small and the right ostium was normal. There was a complete uterine septum extending from uterine fundus to external os with a longitudinal vaginal septum. Only after Hysteroscopy, this diagnosis was confirmed.

For both patients, vaginal septum was excised followed by unification of 2 cervices. Transcervical resection of uterine septum was done with resectoscope. The post-operative period was uneventful. Patients were given estradiol valerate in post-operative period to promote endometrial healing and to prevent intrauterine adhesion formation.

A follow-up hysteroscopy showed a normal uterine cavity.

\section{DIFFERENTIAL DIAGNOSIS}

Complete septate uterus with longitudinal vaginal septum with uterus didelphys.

\section{DISCUSSION}

This unusual Müllerian anomaly is inconsistent with the theory of linear caudal to cephalad Müllerian fusion. ESHRE classification has divided the female genital tract anomalies into uterine, cervical and vaginal anomalies. Uterine anomalies are subdivided into 7 classes:

- U0 Normal uterus.

- U1 Dysmorphic uterus.

- U2 Septate uterus.

- U3 Bicorporeal uterus.

- U4 Hemi-uterus.

- U5 Aplastic.

- U6 Unclassified Malformations.

Among them, the septate uterus is the commonest. These uterine anomalies may be associated with cervical and/or vaginal anomalies. However, complete septate uterus with a cervical septum and longitudinal vaginal septum is a rare uterine anomaly.

Patients described in literature have presented with a variety of obstetric and gynaecologic symptoms, some have been asymptomatic. Diagnosis is made by careful examination and imaging. MRI provides good cervical imaging and is considered the best non-invasive method for 
differentiating septate, bicornuate and didelphys uterus. However, in our cases MRI findings were inconsistent with hysterolaparoscopy findings. 3D TVUS is emerging as a better non-invasive diagnostic tool for Müllerian anomalies.[1] Khaled Abd et al compared the different imaging modalities to differentiate septate and bicornuate uterus.[1] They found sensitivity of $77.4 \%$ and specificity of $60 \%$ for HSG. MRI showed sensitivity of $93.5 \%$ and specificity of $80 \%$. The 3D ultrasound showed the highest diagnostic parameters with sensitivity of $96.7 \%$ and specificity of $100 \%$. However, if the diagnosis is inconclusive with the various imaging modalities, hysteroscopy is the best way to confirm the diagnosis.

Our patients came with infertility and variable findings on USG, HSG and MRI without any conclusive diagnosis. Direct visualisation with hysteroscopy revealed the final diagnosis. It also facilitated to perform the corrective surgery to improve the fertility and future obstetric outcome. Resection of the vaginal septum is easy and commonly performed. Hysteroscopic resection of a uterine septum to improve the obstetric outcome is the gold standard and is indicated in females with unexplained infertility and previous poor obstetric outcomes. Seddigheh et al reported a pregnancy rate of $67 \%$ and a live birth rate of $57.5 \%$ in infertile women who underwent hysteroscopic septoplasty.[2] Kazem Nouri et al reported overall pregnancy rate of $69 \%$ and live birth rate (LBR) was $49 \%$ after hysteroscopic septoplasty.[3]

The union of the two cervices is another area of controversy. Vijay A et al avoided the resection of cervical septum to avoid cervical incompetence in future. ${ }^{[4]}$ However, Meen Jiun Seet et al, SC Ribeiro et al and ME Parsanezahed et al favoured resection of cervical septum.[5,6,7] ME Parsanezahed et al found that resection of cervical septum during Hysteroscopic metroplasty of uterine septum makes the procedure safer, easier and less complicated than the procedure with preservation of the cervical septum.[7] The reproductive outcome is the same in the two procedures. Hence, we also proceeded with unification of cervices.

Most of the recent studies favour non-invasive imaging modalities as the preferred diagnostic tests for Müllerian anomalies. However, in our cases non-invasive imaging modalities gave a misleading diagnosis. Confirmatory diagnosis was made only with hysteroscopy. Hysteroscopy has got a therapeutic value in addition to its diagnostic abilities. Hysteroscopy should be recommended when imaging modalities do not give a confirmative diagnosis, especially in symptomatic patients.

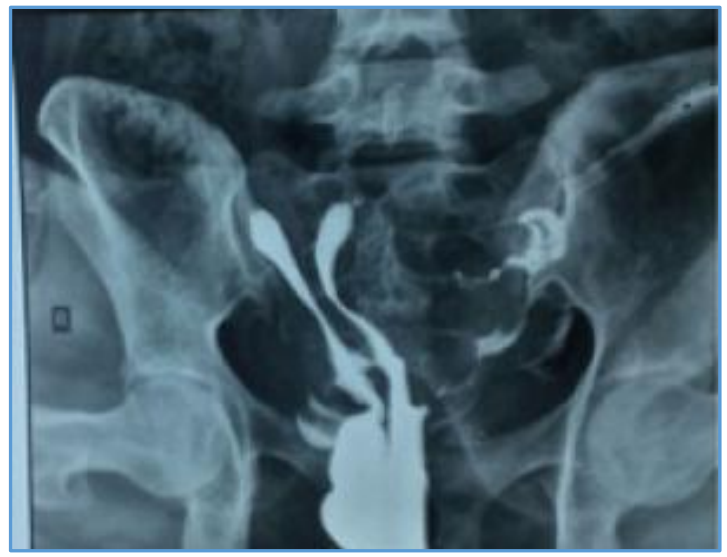

Figure 1. HSG Image showing 2 Uterine Cavities with 2 Cervices

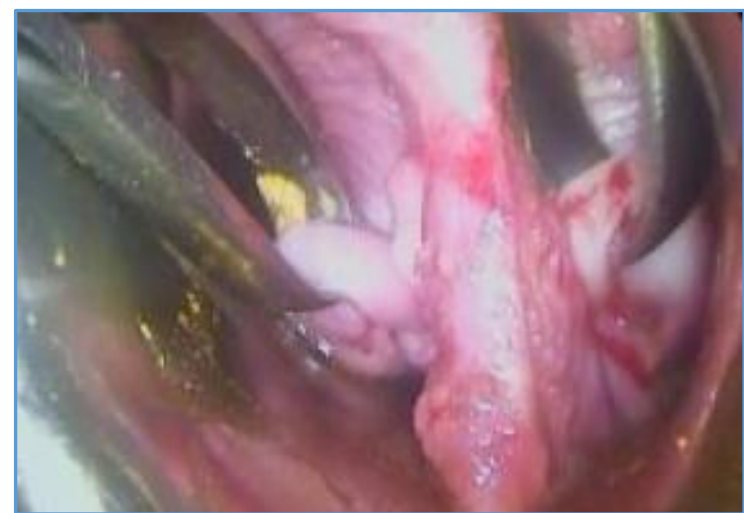

Figure 2. Hysteroscopy Image showing Cervical and Longitudinal Vaginal Septum

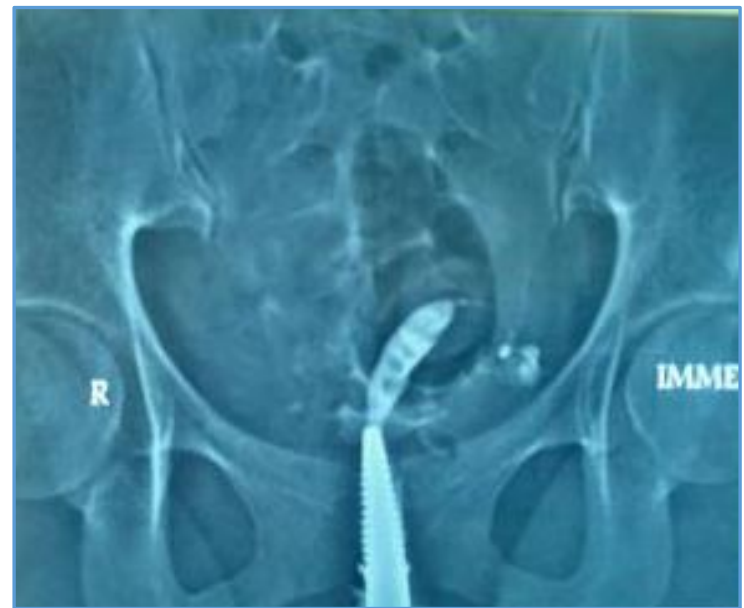

Figure 3. HSG Image showing Left Unicornuate Uterus with Left Tube

\section{FINAL DIAGNOSIS}

Complete Uterine Septum with Longitudinal Vaginal Septum.

\section{REFERENCES}

[1] Khaled AAAD, Mohamed MH, Dina GEE. Septate or bicornuate uterus: accuracy of three-dimensional trans-vaginal ultrasonography and pelvic magnetic resonance imaging. Egypt J Radio Nuc Med 2014;45(3):987-95.

[2] Esmaeilzadeh S, Delavar MA, Andarieh MG. Reproductive outcome following hysteroscopic treatment of uterine septum. Mater Sociomed 2014;26(6):366-71.

[3] Nouri K, Ott J, Huber JC, et al. Reproductive outcome after hysteroscopic septoplasty in patients with septate uterus - a retrospective cohort study and systematic review of the literature. Rep Bio Endocrinol 2010;8:52.

[4] Vijay A, Salve A, Murdia K, et al. An unusual case of septate uterus with double cervix and longitudinal vaginal septum simulating uterus didelphys. Int J Reprod Contracept Obstet Gynecol 2017;6(1):303-5.

[5] Meei JS, Matthew SKL, Jerry KYC, et al. Management of complete vagino-uterine septum in patients seeking fertility: report of two cases and review of literature. Gynecology and Minimally Invasive Therapy 2015;4(4):140-5. 
[6] Ribeiro SC, Yamakami LY, Tormena RA, et al. Septate uterus with cervical duplication and longitudinal vaginal septum. Rev Assoc Med Bras (1992) 2010;56(2):254-6.
[7] Parsanezhad ME, Alborzi S, Zarei A, et al. Hysteroscopic metroplasty of the complete uterine septum, duplicate cervix and vaginal septum. Fertil Steril 2006;85(5):1473-7. 